\title{
VULNERABILITIES OF CAN BUS \& AUTOMOBILE SECURITY
}

\author{
Sahid $\operatorname{Irfan}^{1}$ and R Udayakumar ${ }^{2}$ \\ ${ }^{1}$ Department of Mechanical Engineering, Bits Pilani Dubai Campus, U.A.E \\ ${ }^{2}$ Department of Mechanical Engineering, Bits Pilani Dubai Campus, U.A.E \\ ${ }^{1}$ sahidirfan.v@gmail.com, ${ }^{2}$ udaya@ dubai.bits-pilani.ac.in
}

\begin{abstract}
In an automobile, CAN-bus is the centralized networking system on which Electronic Control Units(ECUs) or nodes broadcast messages or data. Automobile security threats have gone from hypothesis to reality. It is possible for a hacker to remotely control ECUs by means of freeware applications and devices. As a result, the modern automobile presents a broad attack surface and a multitude of potential vulnerabilities to exploit. Security of the CAN-bus can be improved by introducing a monitoring node into the network and using cryptographic techniques. This research highlights the elements of the CAN-bus network, cryptographic methods and suggestions for future development and safety of an automobile.
\end{abstract}

Keywords - CAN-bus, Automobile, Automobile Security, Threat Model, Hack, Vulnerability, Connected system, secured network

\section{INTRODUCTION}

In the developed world, there is arguably no appliance more prevalent in people's lives than the automobile. Growing at an even faster rate is the prevalence of computers in modern automobiles. Computers began to find their way into passenger vehicles in the early 1980s with the advent of the Engine Control Unit (ECU). CAN-bus is the centralized networking system on which Electronic Control Units(ECUs) or nodes broadcast messages or data. By managing basic engine functions, early ECUs brought about improvements in performance and fuel efficiency and also lowering vehicle emissions [1]. Security specialists have exhibited a progression of vulnerabilities in CANbus network. Vulnerabilities of automotive system security vary from a hacker having the ability to trigger a vehicle's horn or lights to seizure of the engine. With the increasing number of interconnections between vehicles, the scope of attack on the internal vehicle networks is rising steeply. Although these networks are shielded against external attacks, they often do not have any foolproof internal security to protect against malicious components or adversaries who can breach the network perimeter. To secure the invehicle network, all communicating components must be authenticated, and only authorized components should be allowed to send and receive messages. This is achieved using an authentication framework. Cryptography is widely used to authenticate communicating parties and provide secure communication channels. As a result, locking down the vehicular systems is the main area of concern for manufacturers and some took up the approach of security by obscurity whereas others sought legal help to silence security researchers and keep vulnerabilities covered. In 2015, Volkswagen engaged a team of European security researchers in a 2-year long legal battle, preventing the group 
from presenting the research paper on a vulnerability they had found in Volkswagen's remote keyless entry system [1].

\section{CONTROLLER AREA NETWORK BUS (CAN-BUS)}

\section{Without CAN}

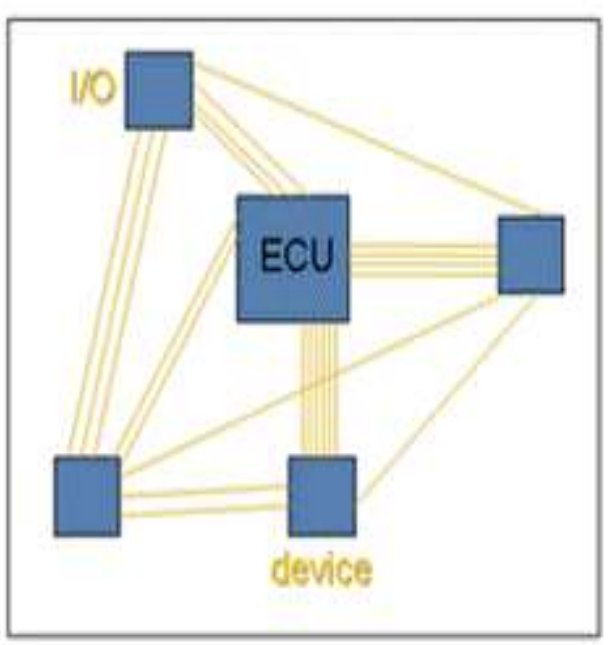

With CAN

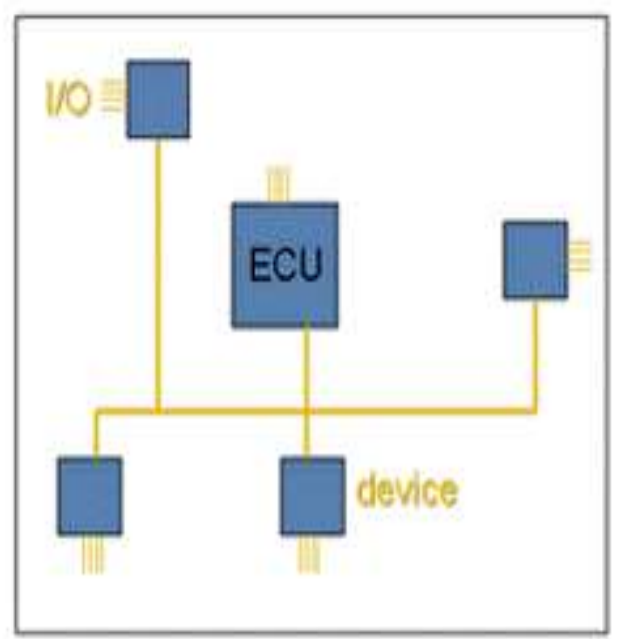

Fig. 1 CAN Network Ergonomically Reduces Wiring

The CAN bus is the workhorse behind most of the existing in-vehicle networks. It was designed by Bosch during 1983 and proved to be so successful ever since that it is present in every car on the market now. CAN-bus is the centralized network, on which vehicular data is being communicated. It carries all the information including the operator commands to the sensor readouts of various components. Development of CAN-bus eliminated the conventional system of point to point contact for any two components to communicate. It increased the overall efficiency and reduced the complexity in addition to the lowered wiring cost. Fig. 1, depicts the advantage of CAN bus structure over the pre-CAN bus architecture i.e., the ECU is placed at the logical center of the pre- CAN network, whereas the involvement of ECU is reduced in the CAN-bus architecture thereby reducing the complexity of the network [1][2].
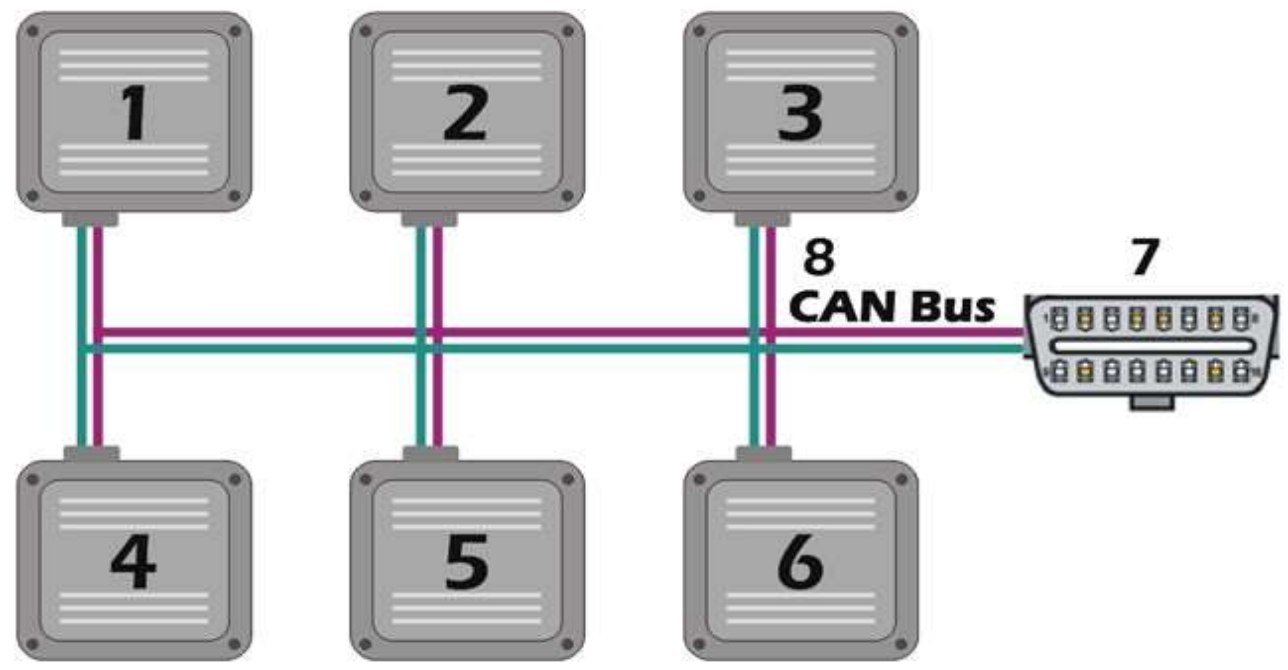

Fig. 2 CAN-bus Schematic Diagram 
In Fig. 2, Nodes numbered 1 to 6 are the different ECU's namely, Engine Management ECU, Transmission ECU, Anti-Lock Braking ECU, Traction Control ECU, Airbag ECU, Power Steering ECU. OBD port is numbered 7 and CAN wires are numbered 8. The wires are called CAN high and CAN low. When the CAN bus is in idle mode, both lines carry $2.5 \mathrm{~V}$. When data bits are being transmitted, the CAN high line goes to $3.75 \mathrm{~V}$ and the CAN low drops to $1.25 \mathrm{~V}$, thereby generating a $2.5 \mathrm{~V}$ differential between the lines. Since communication relies on the voltage differential between the two bus lines, the CAN bus is NOT sensitive to inductive spikes, electrical fields or other noise. This makes CAN bus a reliable choice for networked communications on mobile equipment.

Other advantages of the network include:

- Ensures noise free transmission.

- Works in different electrical environment.

- Eliminated traffic congestion.

- Prioritized data transmission.

- $\quad$ Nodal monitoring of error frames [3].

- Low-Cost, Lightweight Network

- Capabilities to perform error checking on each frame.

\section{VULNERABILITIES OF CAN BUS}

\subsection{LACK OF BOUNDARY PROTECTION \& SEGMENTATION}

All messages are broadcasted over the CAN network, which can be accessed any ECU or node. Interconnectivity between different components rise the primary security issues in the modern automobile. To understand the magnitude of these issues, it is important to to know how these components are interconnected. In Fig. 3 components in blue are related to functioning of the vehicle (e.g., engine management system, brake controllers, etc), components in pink are related to safety (e.g., airbag, seatbelt pre-tensioners, etc), components in yellow are related to entertainment and comfort (e.g., infotainment, telematics unit, etc.,). Every highlighted component here has the potential to be exploited by an attacker. A weakness in any one of these components could grant an attacker access to the rest of the vehicle's systems.

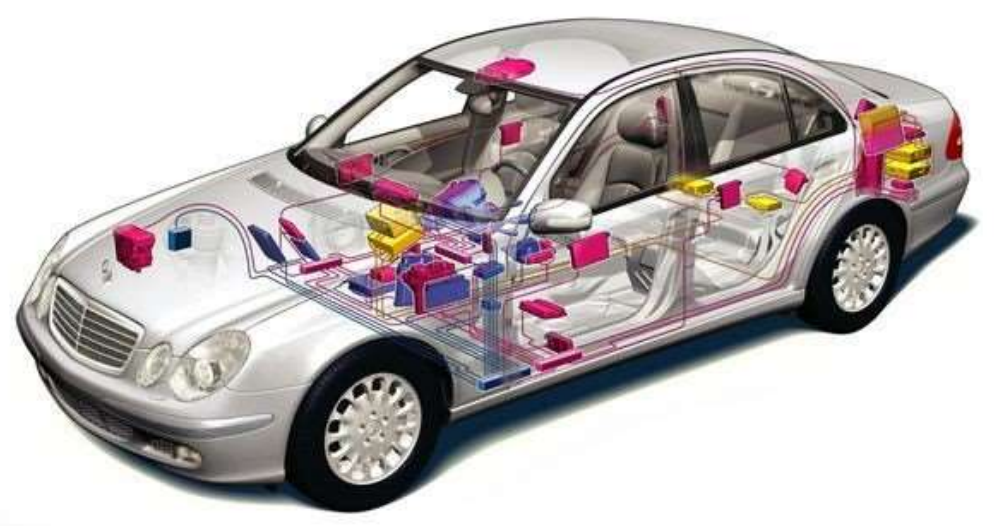

Fig. 3 Interconnected components in a car 


\subsection{LACK OF AUTHENTICATION \& ENCRYPTION}

Many third-party solutions are available to understand the messages broadcasted in the network which help the attackers to study the process. CAN protocol is incapable of distinguishing between a legitimate device and a malicious one. In-addition to that, it does not provide any encryption mechanism to safeguard the integrity and privacy of the data.

\subsection{VULNERABLE TO DENIAL OF SERVICE ATTACKS}

This attack can be performed by transmitting consecutive high priority messages by sending successive dominant bits on the network, i.e., Replacing recessive bits with dominant ones in the arbitration phase would result in the target ending transmission due to arbitration. Such attacks can result in flooding the bus, bringing the network down or occupying all bandwidth which will make other nodes unable to transmit any messages [4].

\subsection{SECURITY BY OBSCURITY}

It is the reliance of the automakers on the privacy of the details of the elements of a system. Appropriately, this model is a failure as it is only a security norm. This is a decision often made in the corporate boardroom. Until malicious vehicle hacking becomes more commonplace, automakers believe it does not warrant a significant amount of budget or attention. However, ignoring vulnerabilities or attempting to hide them does not lead to more secure systems. The only way to overcome this flawed model is through increased public exposure of security vulnerabilities. One of the goals of this paper is to increase awareness of the significant degree to which modern automotive systems are insecure.

\section{AUTOMOTIVE THREAT MODEL}

Threat modeling is the movement of characterizing a hypothetical model of the known dangers to a system. The better the presumptions, the nearer is the hypothetical model to the practical execution to capture the attack vectors.

\subsection{BLUETOOTH}

The Bluetooth interface is also used to connect various carry in devices to vehicles and to provide many valuable functions, for example, mobile-phone connection (voice calling and voice dialing), streaming audio, SYNC address-book/schedules, PC/tablet tethering, car-diagnostics and health-monitoring. As the result, almost all recent vehicles have Bluetooth connection as standard options. Generally, there are two attack scenarios involved. The principal attack scenario is when Bluetooth is active and unpaired, providing the attacker adequate information and time to get into the action. The second method arises when the pairing happens, which is less dangerous as the pairing process takes minimal time [5].

Researchers from the Argus Research Team could stop the engine of moving vehicle using "Just works pairing mechanism" i.e., by connecting to the Bosch Drivelog OBD-II dongle via Bluetooth. Drivelog is Bosch's smart device for collecting and managing your vehicle's operating data. Fig. 4 explains the Drivelog's pairing mechanism, which possessed two vulnerabilities. The first vulnerability, an information leak in the authentication process, between the dongle and the smart phone application allowed them to quickly brute-force the secret PIN offline and connect to the dongle via Bluetooth. After being connected, security holes in the message filter of the dongle allowed them to inject malicious messages into the CAN bus. The physical proximity does not equal 
Bluetooth range. Standard Bluetooth range is about $10 \mathrm{~m}$, but a Bluetooth dongle can be modifiedto10x or 100x more range [6].

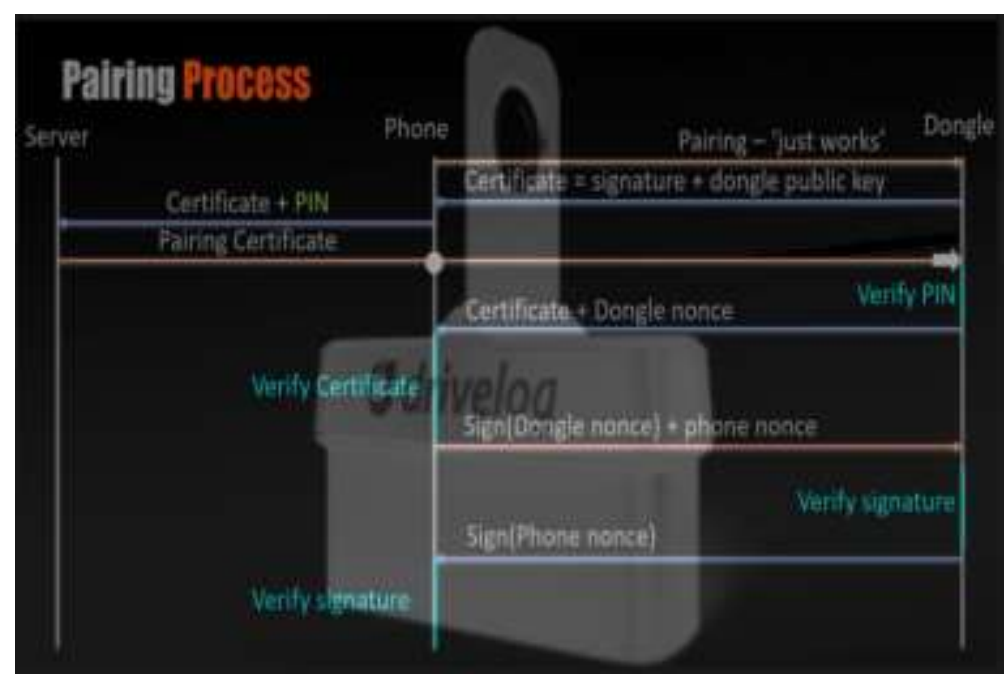

Fig. 4 Drivelog Pairing Mechanism

\subsection{REMOTE KEYLESS ENTRY/START (RKE)}

Car key systems have passed through several generations, evolving from the simple physical keys to more sophisticated keyless entry systems. Key fobs are equipped with a concise wireless transmitter that transmits RF signals to the transceiver of the vehicle. The encoded information received by the transceiver is analyzed by the ECU, which decides whether the key is legitimate and eventually lock, release and start the vehicle. This analyzing ECU is also linked to CAN-bus [7].

4.2.1. ROLLJAM: RollJam is a device that unlocks almost all car's doors, commonly known as "Universal car remote". In Fig. 5, The gadget jams and stores the radio frequency signal broadcasted from the key fob, which can be used to unlock/lock the vehicle. Since the device jams the radio frequency signal, vehicle user may snap again to make sure the vehicle is locked. In this scenario, the gadget broadcasts the primary and jams the later signal. This misleads the user to believe the vehicle is locked. RollJam has a potential remote attacking range of five to twenty meters [7].

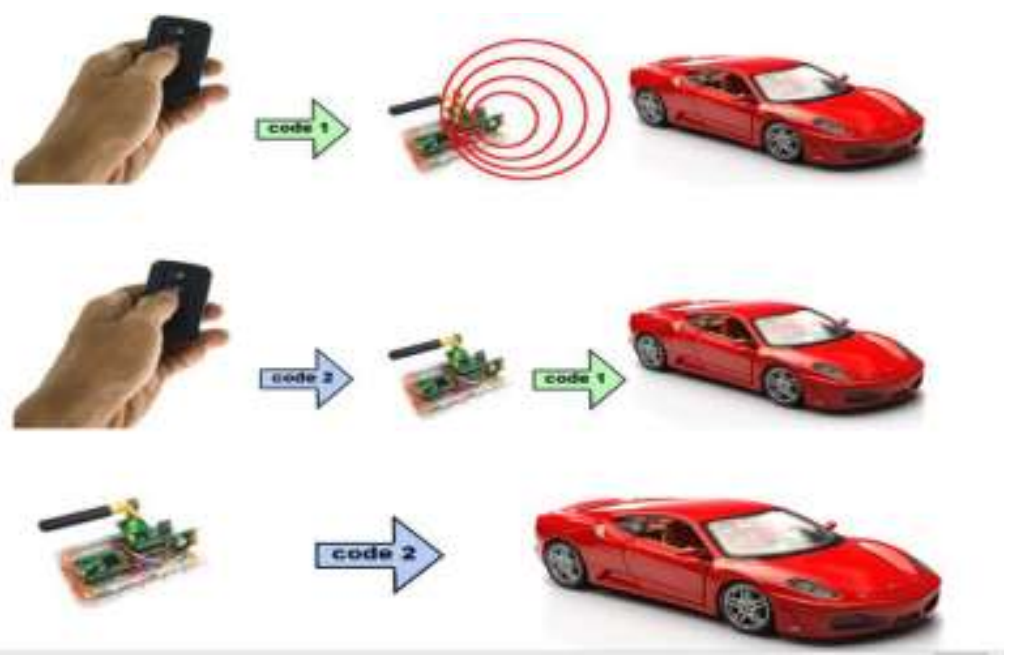

Fig. 5 Functioning of RollJam 


\subsection{TIRE PRESSURE MONITORING SYSTEM (TPMS)}

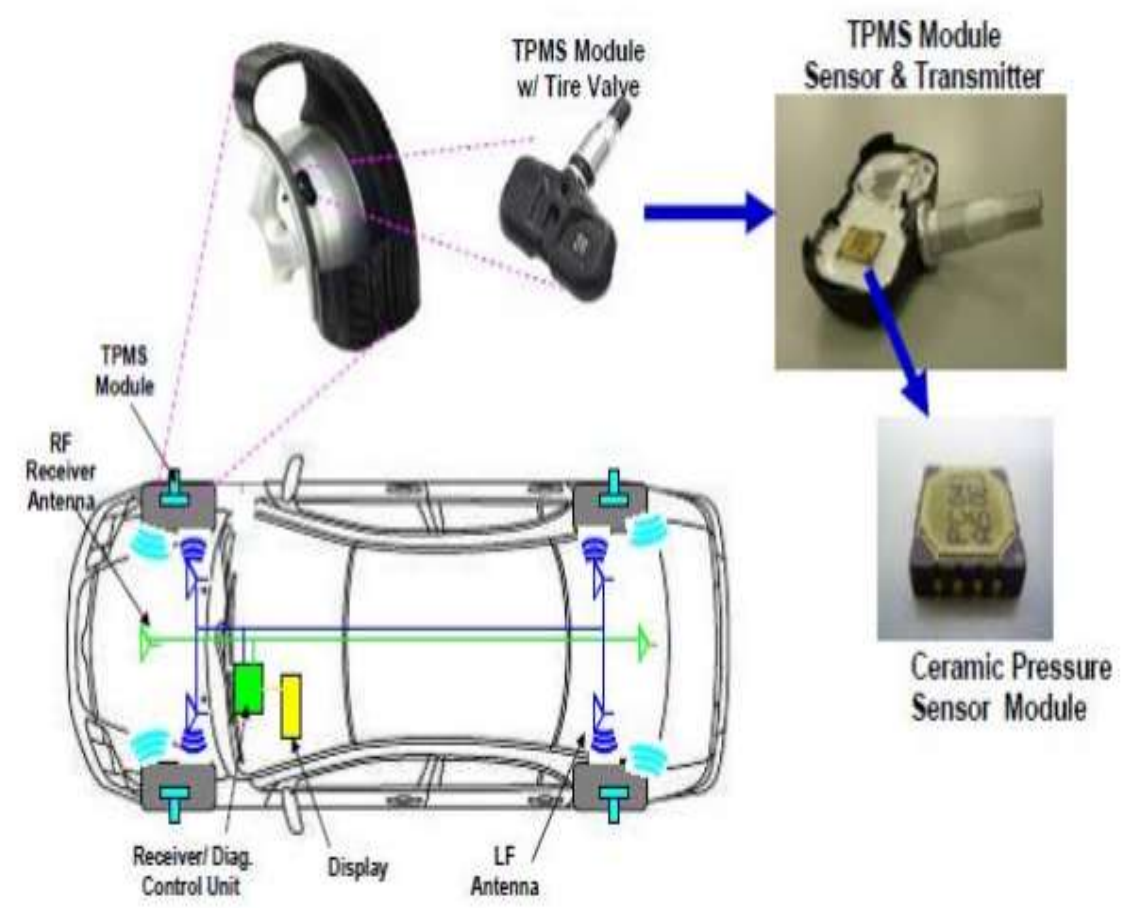

Fig. 6 TPMS Monitoring System

In Fig. 6, the TPMS system typically consists of transmitters in the tires and a receiver within the car. Since a wired connection from a rotating tire to the vehicle's electronic control unit is difficult to implement, the sensor module communicates its data via a radio frequency (RF) transmitter. The receiving tire pressure control unit analyzes the data and can send results or commands to the central car computer over the Controller-area Network to trigger a warning message on the vehicle dashboard The tire pressure sensors contain unique IDs, so merely eavesdropping enabled the researchers from Rutgers University and the University of South Carolina in identifying and tracking vehicles remotely. Beyond this, they could alter and forge the readings to cause warning lights on the dashboard to turn on, or even crash the ECU completely [8]. This attack is possible within a range of 1 meter [9].

\subsection{PASSIVE ANTI-THEFT SYSTEM (PATS)}

It could be possible to create a denial of service attack that could cause the car no longer to start, despite the right key inserted. If remote attacks are involved this attack floor may be very minimal and must be within a range of $10 \mathrm{~m}$. The most effective data transferred is the radio frequency signal and the identification code. It is far difficult to assume a utilizable vulnerability in this code. The principle exploitation vector might be for car robbery.

\subsection{TELEMATICS/WI-FI/CELLULAR}

Modern cars are equipped with a cellular radio, vehicle communicates with the cellular network using this cellular radio. General Motors launched "OnStar" for this purpose. It retrieves traffic data or weather forecasting details. In some cars, it acts as a WiFi hotspot. The telematics system introduced by the Toyota Prius, brings up the provision for emergency communication, vehicle theft tracking, roadside support system [1]. 
Telematics/Wi-Fi/Cellular is the key success factor for the attackers as the range is very large. The immobilization of the car in Austin using WebTeckPlus application by a furious employee in 2010, Charlie Miller \& Chris Valasek's breakthrough in a 2014 model Jeep Cherokee using the communication system Uconnect, Sami Kamkar's OwnStar, which is a gadget and can recognize close by operators of the OnStar RemoteLink application on a cell phone, are some of the best examples that prove telematics are highly potential surface for attackers.

\subsection{OBD-II PORT \& INFOTAINMENT SYSTEM}

The OBD-II serves as a direct interface into the car internal network. Therefore, an adversary with local access can install a working attack device [9]. The car owner may be unintentionally using a counterfeited aftermarket OBD-II device after opting for a lowprice replacement part. That local-access attacks are not limited to the OBD-II diagnostic port. An attacker may attach and hide the attacking device anywhere along the CAN-bus. Think of a malicious repair shop or the installation of rogue replacement parts that require CAN-bus connections for their operation, like aftermarket infotainment units, parking sensors modules, or anti-theft systems. Charlie Miller \& Chris Valasek demonstrated reverse engineering on the vehicles CAN-bus 'and they were able to nonstop blast the horn to hazards like jamming brakes at high speeds. They used a laptop which was connected into the vehicle's 'OBD-II port via ECOM cables.

\section{SECURE CAN-BUS}

\subsection{CONVENTIONAL RESEARCH}

Recently there are many researches being conducted to secure CAN-bus from malicious attacks or more to be specific, from spoofed messages [10]. Even though most of the researches conducted have found solutions, they all face the same problem of implementation at all nodes, which is very complex, time consuming and expensive process. For real-time data processing, these researched systems are intolerable for invehicle control systems. For vehicle applications, simple authentication and key delivery protocols are crucial to eliminate calculation time.

\subsection{INVENTORY OF AUTHORIZED AND UNAUTHORIZED DEVICES}

Establishing a comprehensive inventory of the controllers in the CAN network, is the first step in the device authentication. This inventory should include all local controllers responsible for vehicular functions but would not be expected to include externally connected devices such as cellular phones or diagnostic devices. For the device authentication to work effectively, the CAN identifier field must be encrypted when in transit. Encryption of the CAN identifier field prevents an attacker from being able to intercept and spoof a legitimate CAN device's identifier field [11].

\subsection{SECURE CONFIGURATIONS FOR HARDWARE AND SOFTWARE}

CAN controllers themselves cannot be effectively secured, it is necessary to employ a securely configured central control unit to oversee and manage CAN-bus communications. A CAN controller generally lacks the processing power to screen messages effectively. Therefore, it is necessary to devise a secure vehicle architecture in which the security of the different controllers is centrally managed. Rather than locking down each individual controller, a centrally-located computer can be positioned to oversee all traffic on the network and make real-time security decisions. 


\subsection{LIMITATION AND CONTROL OF NETWORK PORTS, PROTOCOLS, AND SERVICES}

Many ports and services only exist for debugging and diagnostic purposes. In such cases, these ports should be closed and the services disabled prior to the vehicle being sold to a customer. These diagnostic services should only be accessible to service technicians or mechanics when the vehicle is placed into a special diagnostic mode. The diagnostic services and ports should never be open or accessible when a vehicle is being driven on the road. An appropriately positioned software firewall should be utilized on the vehicle's internal network to filter messages originating from the vehicle's Wi-Fi, Bluetooth, or cellular services certainly should not be allowed to pass through to controllers on the internal network[12]

\subsection{KEY-HASHED MESSAGE AUTHENTICATION CODE (HMAC)}

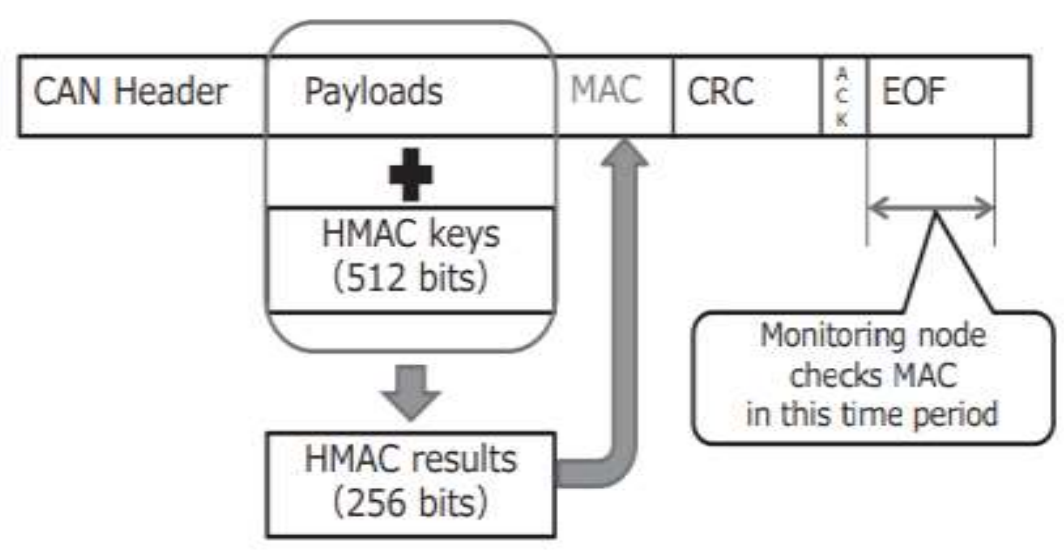

Fig. 7 HMAC calculation timing

Function of HMAC is to prove the integrity of a message over an untrusted network [13] i.e., it makes sure the message has arrived unaltered. It is a code summary of a file having definite size. Any small change in the file data can produce entirely different hash. The application of HMAC can be used in the authentication and encryption of data in the network. In Fig. 7, the MAC is given to a part of the payload to assure the integrity of the CAN message.

\subsection{BOUNDARY DEFENSE THROUGH SEGMENTATION (PROTOTYPE)}

In Fig. 11, CAN network is split into three segments: safety CAN, vehicle control CAN, and infotainment \&comfort CAN. Components can still communicate across different buses but must be authenticated by the monitoring node. The Monitoring Node is the centralized unit which is equipped with an intrusion prevention system (IPS). Preventing the attacker from the penetration of the important segments through any easily accessible entry point. All external interfaces on their own dedicated network segment, preventing direct access to the CAN bus from an external device. 


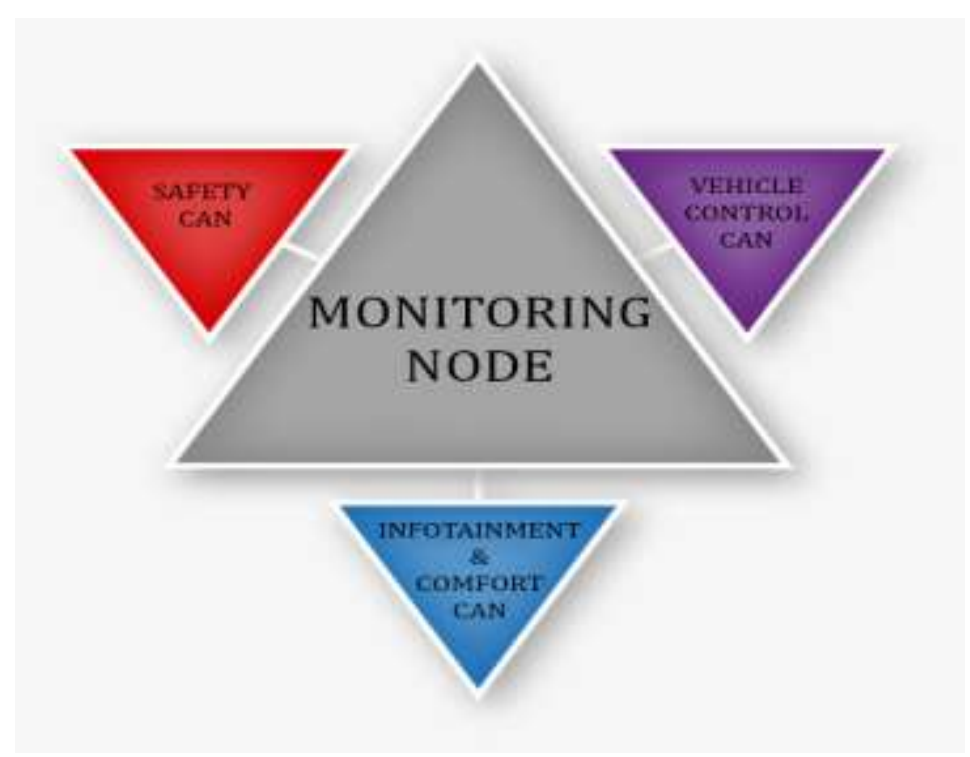

Fig. 11 Schematic representation of the proposed segmented CAN network

\section{SPOOFED MESSAGE MONITORING SYSTEM-(PROTOTYPE)}

As it is very easy for an attacker to spoof messages to the conventional network, securing CAN-bus privacy was a real challenge to manufactures. In Fig. 8, the proposed Spoofed message monitoring system, is designed by adding a monitoring node to the conventional CAN-bus communication system. Monitoring node is just another add-on node to CAN-bus network like any ECU. It consists of two parts, an improved Can controller which monitors the network for any unauthenticated messages. If the controller encounters with an unauthenticated or a spoofed message, it immediately flags the message and overwrites it with an error frame. Each message from an ECU will be equipped with a Message Authentication code(MAC), which is a portion of the encryption key. Encryption key is generated with the help of a programmed randomizer, which allocates each key to the specific data or message which is prepared for broadcasting. This new data will be checked by (Cyclic Redundancy Check) CRC for any errors, which is further broadcasted in the network. The broadcasted message is verified by the monitoring node with the accurate information from the randomizer equipped. Thus the network can be secured from any foreign activity, which challenges the security of the standard Can Bus network.

Basically, this happens in two phases:

1) Authentication of data using MAC at nodes

2) Detection of spoofed messages and overwriting the messages with an error frame.

\subsection{REQUIREMENTS FOR THE IMPLEMENTATION:}

1) An improved Can Controller

2) Updated programs for programming ECU

3) HMAC based randomizer 


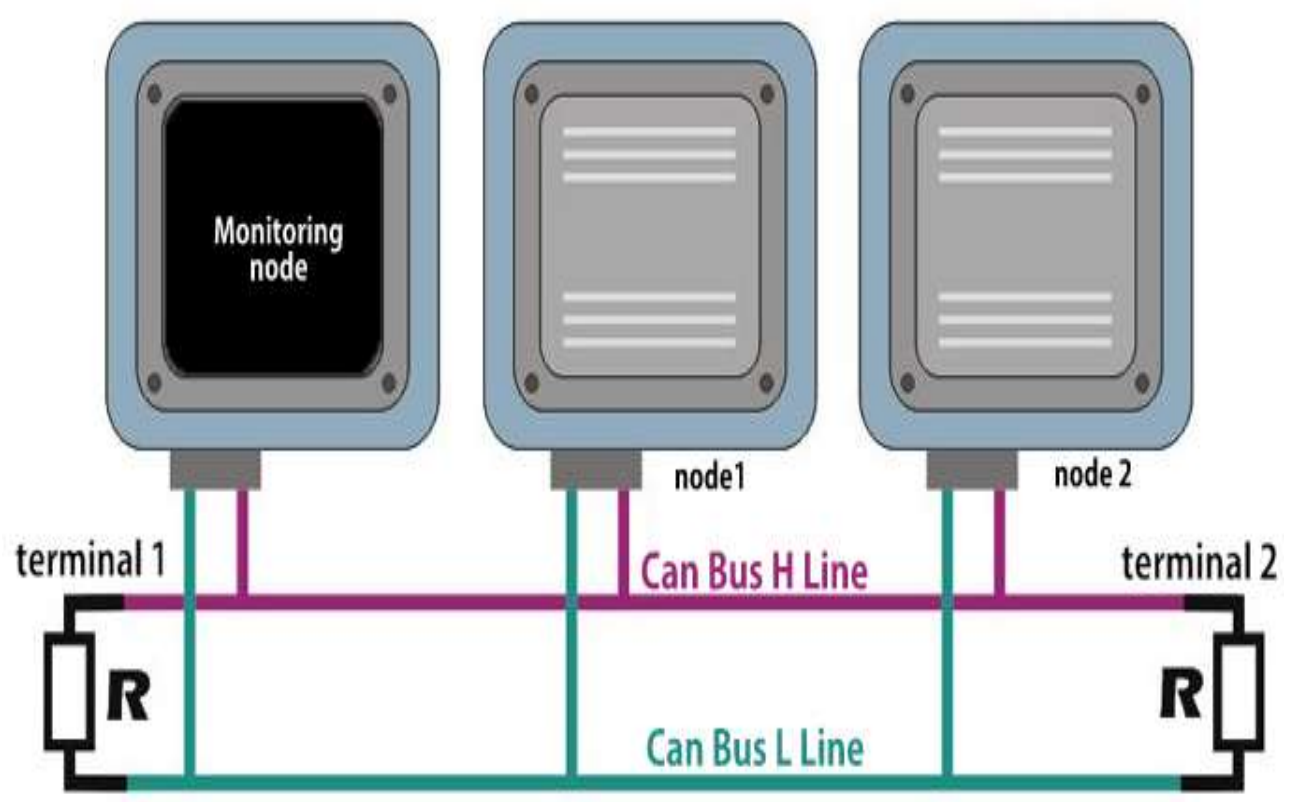

Fig. 8 Spoofed message monitoring system

\subsection{ADVANTAGES}

1) The proposed prototype provides a lucid and secure network solution for the complex issues CAN Bus is vulnerable to. It eliminates the risk of redesigning the conventional CAN Bus for enhancing the security by introducing a monitoring node to the network.

2)The only possible way for any malicious attacker to target the monitoring system is by spoofing messages. As soon as the spoofed message is injected into the network, monitoring node flags it as an error frame. Thereby, it not only looks into the safety of the network but also ensures its own protection, being a message filtering unit between the sender and the receiver node. Considering the safety of the introduction security prototype is another challenge automaker must overcome.

3) Its implementation is easier, as it can be added to the network of the existing automobiles with the help of OBD ports. On the other hand, based on the convenience of the automaker it can be implemented effortlessly as it is similar to any other component in the network.

\section{SECURE REMOTE KEYLESS ENTRY - (PROTOTYPE)}

Usually car's lock/unlock by a single press on the key fob, as the button is pressed microcontroller transmits a chain of 64 or above bits to the key-fob's radio frequency 'transmitter, where the modulation of the chain takes place and is 'radiated through a 'printed-looped 'antenna [14]. The radiated RF signal contains a rolling code along with the fixed code, this rolling code was added for securing the fixed code. Unfortunately, this rolling code even though it keeps changing, is vulnerable to Rolljam. 


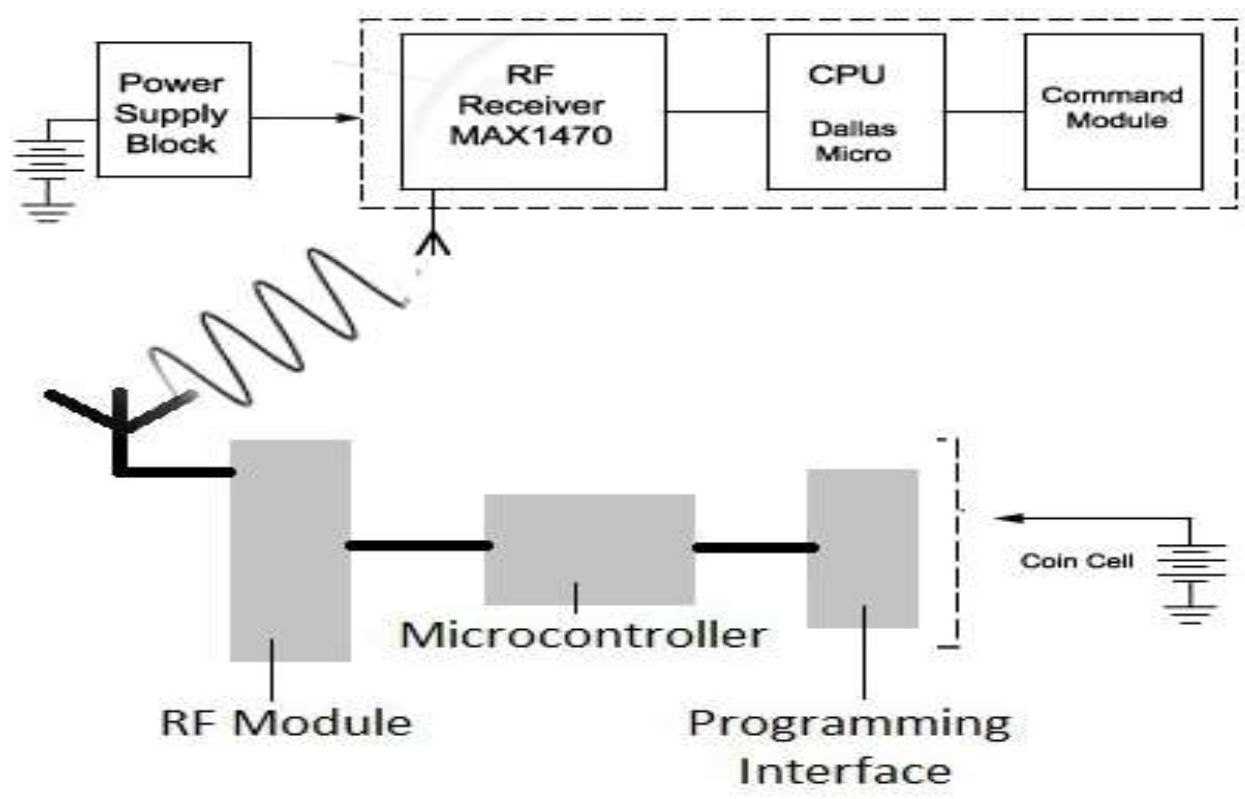

Fig. 9 Proposed Circuit of a RKE \& Car's Transceiver Data Transmission

In Fig. 9, For the proposed system, cars should have board computer, armed with transceivers capable of generating random numbers and communicating with key fob. It is very important to use Random Number Generators (RNGs) because of the rare probability of repeated number sequence for transceiver as well as for key fob.

In Fig.10, Multi level authentication is demonstrated to eliminate the intervention of gadgets like RollJam. When the button is pressed on the key fob, unique id is send to the car's transceiver .Board computer checks the id and send back a generated random number request for authentication. Authentication message build by the key fob is send as a response to the request.As the authenticated message is verified by the board computer car unlocks/locks/opens the boot.

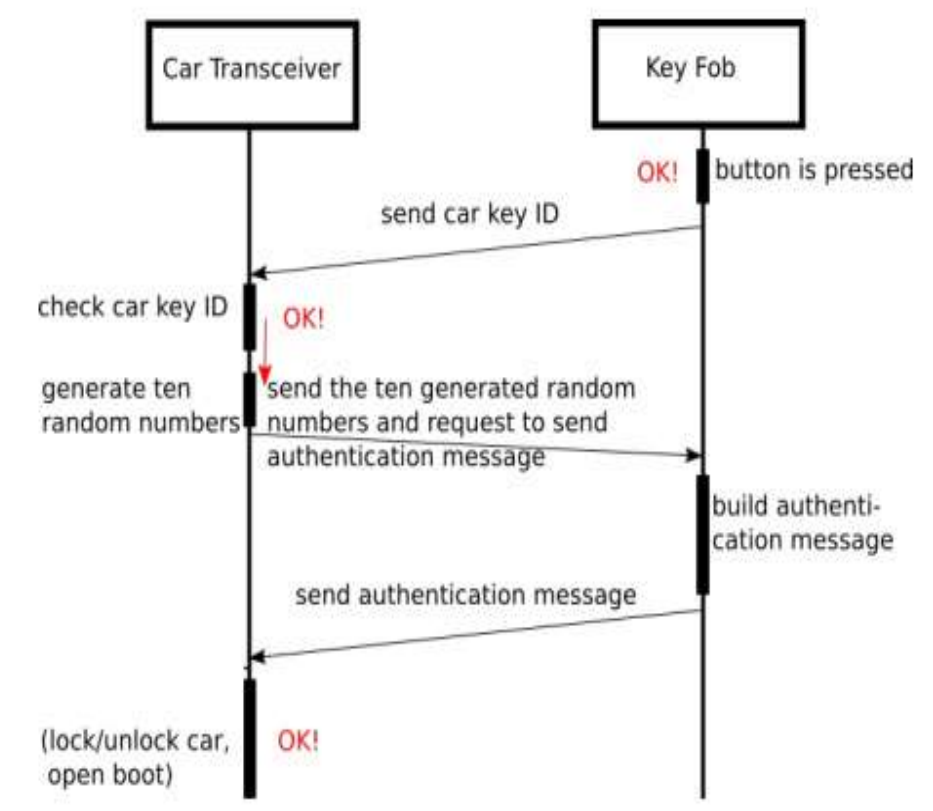

Fig. 10 Schematic diagram of key fob-transceiver communication 


\section{CONCLUSION}

Automobile is now an important factor in common man's life. As technologies have advanced, so should be the security level of automotive. Securing the automotive is equal to securing people's life. Bypassing a car's present security is not a tough task, even an undergraduate school student can do it. Conventional policies of automakers are proven to be less effective and reliable. They must stop fixing up safety issues and begin developing secure networks from the base.

Car hacking can also be done as a type of security audit. By auditing the security of one's own vehicle, it is possible to gain an improved understanding of the ways in which the vehicle might be vulnerable to attack and to take precautions accordingly. Most computer users would not trust a new web browser or a new operating system if they knew it had not undergone extensive penetration testing by the developer. Why, then, do we entrust our safety and the safety of our loved ones to automotive systems that are not audited for security by their manufacturers?

\section{REFERENCES}

[1] Roderick Currie, Manuel Santander "Information Security Reading Room: Manuel Santander "Developments in Car Hacking" (2015).

[2] Tarun Agarwal. "Controller Area Network" (2015).

[3] Robert Buttigieg, Mario Farrugia, Clyde Meli. "Research Gate: Security Issues in Controller Area Network in Automobiles". (2017)

[4] Chris Valasek and Charlie Miller. "IOActive: A Survey of Remote Automotive Attack Surfaces". (2014)

[5] Pedro Umbelino, “Obd-Ii Dongle Attack: Stopping A MovingCar Via Bluetooth Retrieved”. (2018)

[6] SamyKamkar. "Anatomy of the RollJam Wireless Car Hack". (2015)

[7] Peter Bright. "ARS Technica: Cars hacked through wireless tire sensors". (2010)

[8] Charlie Osborne, "OwnStar: Unlock and track any GM OnStar connected car for \$100”.(2015)

[9] Hiroshi Ueda and Ryo Kurachi, "SEI TECHNICAL: Security Authentication System for In-Vehicle Network", (2015).

[10] Maxim. "Maxim Integrated: Requirements of Remote Keyless Entry (RKE) Systems". (2015)

[11] Roderick Currie. "Information Security Reading Room: The Automotive Top 5: Applying the Critical Controls to the Modern Automobile" (2016).

[12] Professor Alexander Wyglinski. “TPMS Receiver Hacking”. (2015)

[13] Hirofumi Onishi. "International Journal of Automotive Engineering: In. Approaches for Vehicle CyberSecurity in the US,". (2017) 